Grażyna Odrowąż-Sypniewska1, Magdalena Krintus ${ }^{1}$, Joanna Siódmiak ${ }^{1}$, Iwona Jaszowska², Radosława Staszak-Kowalska ${ }^{2}$, Anna Zawadzka-Krajewska ${ }^{3}$, Szymon Kierat $^{3}$, Urszula Demkow ${ }^{4}$

${ }^{1}$ Department of Laboratory Medicine, Nicolaus Copernicus University Collegium Medicum, Bydgoszcz, Poland

2Department of Pediatrics, Pneumonology and Allergology, Children's Hospital, Bydgoszcz, Poland

${ }^{3}$ Department of Pediatrics, Pneumonology and Allergology Medical University of Warsaw, Poland

${ }^{4}$ Department of Laboratory Diagnostics and Clinical Immunology, Medical University, Warsaw, Poland

\title{
Serum 25(OH)D status and lipid profile in children with newly diagnosed asthma
}

\author{
Corresponding author: \\ Grażyna Odrowąż-Sypniewska, PhD, Prof \\ Department of Laboratory Medicine \\ Collegium Medicum, Nicolaus \\ Copernicus University \\ Sklodowskiej-Curie No. 9, 85-094 By- \\ dgoszcz, Poland \\ Phone + 48525854046 \\ Fax +48525853603 \\ E-mail: odes@cm.umk.pl
}

\begin{abstract}
Background. The problem of the influence of hyperlipidemia on asthma was addressed several years ago Systematic review and meta-analysis performed in the pediatric population on the association between vitamin D status and lipid profile components revealed discordant results and indicated that higher serum $25(\mathrm{OH}) \mathrm{D}$ is related to a more favorable lipid profile.

Objective. We aimed to elucidate whether there was an association between vitamin D status and lipid profile components and apolipoprotein B in a sample of children with newly diagnosed atopic asthma. Methods. The study included 150 children aged 2-12 years. Atopic asthma was diagnosed in 110 children; 40 children constituted a reference group. Fasting blood was collected to measure 25(OH)D total, lipid profile and apolipoprotein B concentrations.

Results. Children with asthma had significantly lower 25(OH)D $(\mathrm{p}<0.0001)$ but similar lipid and apolipoprotein B concentrations. The proportions of hypercholesterolemia, hypertriglyceridemia and increased apoB concentrations were similar in both groups. HDL-C concentrations in asthmatic 25(OH)D-deficient children were higher compared with the children with sufficient levels $(p=0.05)$. ApoB concentration was lower in $25(\mathrm{OH}) \mathrm{D}$-deficient compared with vitamin D sufficient asthmatics $(\mathrm{p}=0.0008)$. Correlations between $25(\mathrm{OH}) \mathrm{D}$ concentration and lipids and apoB in asthmatics revealed gender differences. An inverse relationships between vitamin $D$ and total cholesterol and HDL-C $(R=-0.39, p<0.05 ; R=-0.475, p<0.001)$ were found in girls. In boys vitamin $D$ correlated with LDL-C and apoB $(R=0.376, R=0.498 ; p<0.001)$. Conclusion. In children with asthma lower $25(\mathrm{OH}) \mathrm{D}$ had more favorable gender-dependent effect on the lipid profile. The association of serum 25(OH)D and lipid levels in children with asthma remains for further studies Key words: 25-hydroxyvitamin D, asthma, lipids, apolipoproteins
\end{abstract}

Folia Medica Copernicana 2015; 3 (3): 113-116

\section{Introduction}

A recent systematic review and meta-analysis performed in the pediatric population on the association between vitamin $\mathrm{D}$ status and lipid profile components have indicated that higher serum $25(\mathrm{OH}) \mathrm{D}$ is related to a more favorable lipid profile [1]. In a nationwide study in the Middle East and North Africa, performed in the pediatric population, a weak but significant inverse relationship of 25(OH)D with blood pressure, total cholesterol (TC), and LDL-cholesterol (LDL-C) was found. The association of vitamin $D$ with HDL-cholesterol (HDL-C) was significantly positive [2]. Another earlier study reported that low serum $25(\mathrm{OH}) \mathrm{D}$ in children was associated with high triglyceride (TG) levels [3]. Similar findings were reported in obese adults, showing $25(\mathrm{OH})$ $D$ deficiency related to lower HDL-C and higher TG [4]. On the other hand, the associations in children were different. Delvin et al. reported no association in boys but the positive relationship between $25(\mathrm{OH}) \mathrm{D}$ and total cholesterol, triglycerides and apolipoproteins $A 1$ and $B$ in girls [4].

The problem of the influence of hyperlipidemia on asthma was addressed by Al-Shawwa et al. nearly 10 years ago [5]. In this study, the effect of serum cholesterol concentration on asthma frequency in children and adolescents was evaluated. It was found that hypercholesterolemia, independently of obesity, 
increased the probability of asthma. A recent study in children with asthma has shown that higher LDL-cholesterol levels were associated with asthma occurrence and this association was amplified by overweight and obesity [6]. This finding is inconsistent with the earlier data indicating no association of apoB concentration with symptoms of asthma or allergy [7]. Having these controversies in mind, we aimed to elucidate whether there was an association between vitamin $D$ status and lipid profile components and apolipoprotein B in a sample of children with newly diagnosed atopic asthma.

\section{Methods}

\section{Study participants}

The study included 110 children aged $2-12$ years referring to the Department of Pediatric Pulmonology and Allergology at the Medical University (Warsaw, Poland) due to asthma-like symptoms. The subjects were recruited from October until April in the years 2012 and 2013. The written informed parental consent for each participant was obtained and the study protocol was approved by the Bioethics Committee at the Collegium Medicum of the Nicolaus Copernicus University. The diagnosis or exclusion of asthma were performed by an experienced pediatric pulmonologist as described previously [8]. All children included in the study did not receive any additional vitamin $\mathrm{D}$ supplementation beyond their regular dietary intake.

Atopic asthma was diagnosed in 110 children. The reference group included 40 children referring to the Outpatient Clinic for a routine check-up; in those children asthma and allergy were excluded by an experienced pediatrician on the basis of their medical history. Children who had any underlying liver, kidney, endocrine diseases or were on drugs/supplements that might affect vitamin $D$ levels were excluded from the study. In all subjects body mass index (BMI) was calculated. Overweight or obesity were established on the basis of BMI-for-age percentiles for girls or boys [9]. Children with BMI between $85^{\text {th }}-95^{\text {th }}$ percentile were considered overweight, while those with $\mathrm{BMI}>95^{\text {th }}$ percentile were considered obese.

\section{Sample collection and laboratory measurements}

Fasting blood was collected in the early morning (7.00-9.00 a.m.). Serum was obtained within $1 \mathrm{~h}$ and stored deep-frozen $\left(-80^{\circ} \mathrm{C}\right)$ until assayed. Serum total cholesterol (TC), low-density lipoprotein-cholesterol (LDL-C), high-density lipoprotein-cholesterol (HDL-C), triglycerides (TG), apolipoprotein B (PENTRA 400, Horiba $A B X$, Montpellier, France) and $25(\mathrm{OH}) \mathrm{D}$ total (EIA, IDS Ltd, Boldon, UK) were measured. 25-hydroxyvita- min D EIA test kit, used in this study, is aligned with the ID-LC-/MS/MS Reference Measurement Procedure. The detection limit for 25(OH)D was $2.5 \mathrm{ng} / \mathrm{mL}$.

We accepted the following cut-off values for normal lipids and apolipoprotein $\mathrm{B}$ : $\mathrm{TC} \leq 170 \mathrm{mg} / \mathrm{dL}$, $\mathrm{HDL}-\mathrm{C} \geq 45 \mathrm{mg} / \mathrm{dL}, \mathrm{LDL}-\mathrm{C} \leq 110 \mathrm{mg} / \mathrm{dL}, \mathrm{TG} \leq 75 \mathrm{mg} / \mathrm{dL}$ up to 9 years of age, $\leq 90 \mathrm{mg} / \mathrm{dL}$ from 10 years of age, apoB < $0.75 \mathrm{~g} / \mathrm{L}$ [4]. Serum 25(OH)D concentration $\geq 30 \mathrm{ng} / \mathrm{mL}$ was accepted as sufficient, $21-29 \mathrm{ng} / \mathrm{mL}$ as insufficient and $\leq 20 \mathrm{ng} / \mathrm{mL}$ as deficient, according to the recommendations for the Polish population [10].

\section{Statistical analysis}

We used descriptive statistics to obtain the characteristics of the study and reference groups. Continuous variables were expressed as mean \pm standard deviation (Gaussian distribution) and median with $25^{\text {th }}$ and $75^{\text {th }}$ percentiles (non-Gaussian distribution). The data were compared using the Student's t-test for normally distributed variables and the Mann-Whitney U-test for non-normally distributed variables. The Spearman or ANOVA correlations were used to analyze the associations between variables. A p value $<0.05$ was considered statistically significant. The statistical analysis was performed using Statistica 10.0 for Windows (StatSoft, Tulsa, OK, USA) and MedCalc 12.7.0 (MedCalc Software, Ostend, Belgium).

\section{Results}

Serum 25(OH)D was significantly lower in children with atopic asthma compared with the reference group, however, vitamin $D$ deficiency was not a characteristic feature of asthmatics. The mean concentrations of lipids and apolipoprotein B concentrations were similar in both groups. The main features in children with asthma and the reference group were borderline $(0.75-<1.0 \mathrm{~g} / \mathrm{L})$ or unfavorable $(\geq 1.0 \mathrm{~g} / \mathrm{L})$ apoB concentration (58\% and 66\%) and hypercholesterolemia (46.8\% and $51 \%$, respectively) (Tab. 1).

The comparison of median lipid and apoB values in relation to $25(\mathrm{OH}) \mathrm{D}$ concentration in asthmatic children is presented in Table 2. We found significantly higher HDL-C concentrations in 25(OH)D-deficient children compared with those with sufficient levels $(p=0.05)$. ApoB concentration was significantly lower in 25(OH)D-deficient compared with vitamin $\mathrm{D}$ sufficient children ( $p=0.0008)$.

Bivariate correlation analysis between $25(\mathrm{OH}) \mathrm{D}$ concentration and lipids and $a p o B$ in asthmatics revealed gender differences. We found weak but significant inverse relationships between vitamin $D$ and total cholesterol and HDL-C $(R=-0.39, p<0.05 ; R=-0.475$, 
Table 1. Biochemical and clinical characteristics of the study participants

\begin{tabular}{lccc}
\hline Variable & Atopic asthma $(\mathbf{n}=\mathbf{1 1 0})$ & Reference group $(\mathbf{n}=\mathbf{4 0})$ & p value \\
\hline Gender M/F (\%) & $53.4 / 46.6$ & $57.1 / 42.9$ & 0.600 \\
Age (years) & $5.0(4.0-7.5)$ & $4.5(3.8-7.0)$ & 0.13 \\
$25(\mathrm{OH}) \mathrm{D}$ [ng/mL] & $27.7 \pm 8.3$ & $35.4 \pm 13.04$ & $<0.0001$ \\
$25(\mathrm{OH}) \mathrm{D} \leq 20 \mathrm{ng} / \mathrm{mL}$ & 18.2 & 10.0 & 0.127 \\
Overweight/obesity (\%) & $10.0 / 11.2$ & $2.1 / 2.0$ & $0.04 / 0.04$ \\
Total cholesterol [mg/dL] & $171 \pm 33$ & $175 \pm 28$ & 0.42 \\
TC $>170 \mathrm{mg} / \mathrm{dL}(\%)$ & 46.1 & 51.0 & 0.836 \\
LDL-C [mg/dL] & $101 \pm 28$ & $108 \pm 24$ & 0.16 \\
LDL-C > 110 mg/dL (\%) & 33.7 & 36.7 & 0.560 \\
HDL-C [mg/dL] & $54 \pm 12$ & $54 \pm 0$ & 0.73 \\
HDL-C < 45 mg/dL (\%) & 21.3 & 18.4 & 0.326 \\
TG [mg/dL] & $71 \pm 40$ & $74 \pm 31$ & 0.73 \\
Hypertriglyceridemia (\%) & 28.1 & 42.9 & 0.06 \\
ApoB [g/L] & $0.83 \pm 0.17$ & $0.85 \pm 0.20$ & 0.55 \\
ApoB > 0.75 g/L (\%) & 58.0 & 66.0 & 0.56
\end{tabular}

BMI — body mass index; 25(OH)D - 25-hydroxyvitamin D; TC — total cholesterol; LDL-C — low-density lipoprotein cholesterol; HDL-C — high-density lipoprotein cholesterol; apoB - apolipoprotein B

Table 2. The comparison of selected biochemical and anthropometric indices in relation to $25(\mathrm{OH}) \mathrm{D}$ concentration in children with atopic asthma

\begin{tabular}{|c|c|c|c|c|c|}
\hline Variable & $\begin{array}{c}25(\mathrm{OH}) \mathrm{D} \\
\text { deficiency } \\
(\leq 20 \mathrm{ng} / \mathrm{mL})^{A}\end{array}$ & $\begin{array}{c}25(\mathrm{OH}) \mathrm{D} \\
\text { insufficiency } \\
21-29(\mathrm{ng} / \mathrm{mL})^{\mathrm{B}}\end{array}$ & $\begin{array}{c}25(\mathrm{OH}) \mathrm{D} \\
\text { sufficiency } \\
(\geq 30 \mathrm{ng} / \mathrm{mL})^{\mathrm{C}}\end{array}$ & $p$ value & Post hoc \\
\hline Age (years) & $6.0(5.0-8.5)$ & $6.0(4.0-7.0)$ & $4.5(4.0-7.0)$ & 0.007 & A v. $C, p=0.002$ \\
\hline BMI $\left[\mathrm{kg} / \mathrm{m}^{2}\right]$ & $15.6(14.6-16.0)$ & $16.1(15.2-17.4)$ & $16.0(15.0-17.0)$ & 0.019 & A v. $C, p=0.019$ \\
\hline $25(\mathrm{OH}) \mathrm{D}[\mathrm{ng} / \mathrm{mL}]$ & $17.3 \pm 2.4$ & $24.2 \pm 2.6$ & $36.8 \pm 5.4$ & $<0.0001$ & $\begin{array}{c}\text { A v. B; A v. C; B v. C } \\
p<0.0001\end{array}$ \\
\hline $\mathrm{TC}[\mathrm{mg} / \mathrm{dL}]$ & $158(147-164)$ & $156(134-174)$ & $153(137-170)$ & 0.925 & - \\
\hline LDL-C [mg/dL] & $86(76-100)$ & $93.5(79-106)$ & $100(80-128)$ & 0.137 & - \\
\hline $\mathrm{HDL}-\mathrm{C}[\mathrm{mg} / \mathrm{dL}]$ & $58(56-66)$ & $56(52-66)$ & $53(48-62)$ & 0.048 & A v. $C, p=0.050$ \\
\hline $\mathrm{TG}[\mathrm{mg} / \mathrm{dL}]$ & $55(45-68)$ & $58(47-70)$ & $55(45-68)$ & 0.432 & - \\
\hline ApoB $[g / L]$ & $0.72(0.68-0.76)$ & $0.82(0.71-0.93)$ & $0.87(0.75-1.03)$ & 0.008 & $\begin{array}{c}\text { A v. } C, p=0.0008 \\
\text { A v. } B, p=0.09\end{array}$ \\
\hline
\end{tabular}

A — children with $25(\mathrm{OH}) \mathrm{D}<20 \mathrm{ng} / \mathrm{mL}$; B — children with $25(\mathrm{OH}) \mathrm{D} 20$ to $\leq 30.0 \mathrm{ng} / \mathrm{mL} ; \mathrm{C}$ - children with $25(\mathrm{OH}) \mathrm{D}>30 \mathrm{ng} / \mathrm{mL} ; 25(\mathrm{OH}) \mathrm{D}-$ 25-hydroxyvitamin D; ApoB — apolipoprotein B; BMI — body mass index; HDL-C — high-density lipoprotein cholesterol; LDL-C — low-density lipoprotein cholesterol; TC — total cholesterol; TG — triglycerides

$\mathrm{p}<0.001)$ in girls. In boys vitamin D correlated with LDL-C and apoB and these relationships were positive $(R=0.376, R=0.498 ; p<0.001)$.

\section{Discussion}

Vitamin D status and its association with lipids in children has not been extensively studied yet and the findings are controversial. Similarly, the associations of asthma with hyperlipidemia were not only diverse but also were affected by the presence of overweight and obesity. The underlying mechanisms still remain to be elucidated. We evaluated the association between vitamin $D$ status and lipid profile components and apolipoprotein B in a sample of children with newly diagnosed atopic asthma.

Unexpectedly, we found that vitamin D-deficient asthmatic children had a more favorable lipid profile 
(significantly higher HDL-cholesterol and lower apoB levels) than children with vitamin D sufficiency. Median value of LDL-cholesterol was also lower in $25(\mathrm{OH}) \mathrm{D}$ -deficient children but the difference did not reach statistical significance. These findings were supported by significant positive correlations of vitamin $D$ with LDL-cholesterol and apoB concentrations observed in boys and a significant inverse relationship of vitamin $D$ with HDL-cholesterol observed in girls.

Our findings are inconsistent with some recent data based on the systematic review and meta-analysis performed on the association of vitamin $D$ with lipids in the pediatric population [1]. On the other hand, they are similar to the data reported earlier by Delvin et al. [4]. We have observed, however, the positive associations of vitamin $D$ with lipids and apoB in boys, whereas they have found no associations in boys but only in girls [4]. They suggested that such gender differences in lipid metabolism may be due to the different effects of sex hormones [4, 11]. In fact, Delvin et al. included the French-Canadian children and adolescents in their study of whom more than $60 \%$ were over 13 years old, whereas in our study, the children were much younger and less than $20 \%$ were in the pubertal stage (girls 8-12 years, boys 10-12 years old).

In a recent study of Argentinean children, severe vitamin $\mathrm{D}$ deficiency was shown to be associated with an increased serum apoB concentration [12]. These findings do not match the results of this study, in which lower apoB was observed in 25(OH)D-deficient children. Notably, the children in our study showed only very low percentage presented with severe vitamin $D$ deficiency $<15 \mathrm{ng} / \mathrm{mL}$ (6.4\%), whereas in the Argentinian population severe vitamin $D$ deficiency was found in almost $79 \%$ of cases. The results of our own study are consistent with those recently reported by Ashraf et al., which suggested a possible role of vitamin D on apolipoprotein synthesis [13], and with those published earlier by Teramoto et al. explaining the binding of vitamin $D$ to LDL particles [14].

In children with asthma higher LDL-C levels were associated with the occurrence of asthma and this association was amplified by overweight and obesity [6]. Among children with asthma in our study the percentage of overweight and obesity was $21.2 \%$, which could affect lipid metabolism only to a minor extent. It is known that the excess body fat leads to the increased storage of vitamin $D$ in the adipose tissue and reduces it's serum concentration and availability. However, it was shown that although serum $25(\mathrm{OH}) \mathrm{D}$ levels may be lower, the concentration of bioavailable one may not be low due to a lower concentration of vitamin $D$ binding protein (VDBP) [15]. We did not measure the concentration of
VDBP and this, besides the limited number of participants, constitutes a limitation of our study.

Finally, we conclude that in children with newly diagnosed yet untreated atopic asthma lower 25(OH)D had more favorable effect on the lipid profile and this effect was gender-dependent. However, the association of serum $25(\mathrm{OH}) \mathrm{D}$ and lipid levels and the underlying mechanisms in children with asthma are not clear and remain for further studies.

\section{Acknowledgements}

We would like to thank children and their parents for the participation in our study. We would also like to thank Gabriele Fulgheri MSc, PhD student in our Department for help in collecting blood samples and demographic data of study participants.

\section{References}

1. Kelishadi R, Farajzadegan Z, Bahreynian M. Association between vitamin D status and lipid profile in children and adolescents: a systematic review and meta-analysis. Int J Food Sci Nutr 2014; 65: 404-410.

2. Kelishadi R, Ardalan G, Motlagh ME et al. National report on the association of serum vitamin $D$ with cardiometabolic risk factors in the pediatric population of the Middle East and North Africa (MENA): the CASPIAN-III Study. Nutrition 2014; 30: 33-38.

3. Rodríguez-Rodríguez E, Ortega RM, González-Rodríguez LG, López-Sobaler AM. Vitamin D deficiency is an independent predictor of elevated triglycerides in Spanish school children. Eur J Nutrition 2011; 50: 373-378.

4. Delvin $\mathrm{E}$, Lambert M, Levy E et al. Vitamin D status is modestly associated with glycemia and indicators of lipid metabolism in French-Canadian children and adolescents. J Nutr 2010; 140: 987-991.

5. Al-Shawwa B, Al-Huniti N, Titus G, Abu-Hasan M. Hypercholesterolemia is a potential risk factor for asthma. J Asthma 2006; 43: 231-233.

6. Chen YC, Tung KY, Tsai $\mathrm{CH}$ et al. Lipid profiles in children with and without asthma: interaction of asthma and obesity on hyperlipidemia. Diabet Metab Syndr 2013; 7: 20-25.

7. Nagel G, Weiland SK, Rapp K et al. Association of apolipoproteins with symptoms of asthma and atopy among schoolchildren. Int Arch Allergy Immunol 2009; 149: 259-266.

8. Odroważ-Sypniewska G, Fulgheri G, Siodmiak J et al. 25-hydroxyvitamin $D$ insufficiency in children with newly diagnosed asthma. Folia Med Copernicana, 2014; 2: 93-97.

9. Kulaga Z, Rozdzynska A, Palczewska I et al. Percentile charts of height, body mass and body mass index in children and adolescents in Poland - results of the OLAF study. Stand Med 2010; 7: 690-700.

10. Karczmarewicz E, Czekuc-Kryskiewicz E, Pludowski P. Effect of vitamin D status on pharmacological treatment efficiency. Dermato-Endocrinology 2013; 5: 299-304.

11. Mittendorfer B. Sexual dimorphism in human lipid metabolism. J Nutr 2005; 135: 681-686.

12. Hirschler $\mathrm{V}$, Maccallini $\mathrm{G}$, Molinari $\mathrm{C}$ et al. Association between vitamin $D$ and Apo B concentrations in Argentinean Indian children. Clin Chim Acta 2014; 429: 147-151.

13. Ashraf AP, Alvarez JA, Gower BA, et al. Association of serum 25-Hydroxyvitamin $D$ and components of the metabolic syndrome in obese adolescent females. Obesity 2011; 19: 2214-2221.

14. Teramoto $\mathrm{T}$, Endo $\mathrm{K}$, Ikeda $\mathrm{K}$ et al. Binding of vitamin $\mathrm{D}$ to low-density-lipoprotein (LDL) and LDL receptor-mediated pathway into cells. Biochem Biophys Res Commun 1995; 215: 199-204.

15. Powe CE, Evans MK, Wenger $\mathrm{J}$ et al. Vitamin D -binding protein and vitamin D status of black Americans and white Americans. N Engl J Med 2013; 369: 1991-2000. 